\title{
The salt-, pH- and oxidant-responsive pervaporation behaviors of weak polyelectrolyte multilayer membranes
}

\author{
Naixin Wang ${ }^{\mathrm{a}}$, Guojun Zhang ${ }^{\mathrm{a}, *}$, Shulan Ji ${ }^{\mathrm{a}, *}$, Zhenping Qin ${ }^{\mathrm{a}}$, Zhongzhou Liu ${ }^{\mathrm{b}}$ \\ a Center for Membrane Technology, College of Environmental and Energy Engineering, Beijing University of Technology, Beijing 100124, PR China \\ ${ }^{\mathrm{b}}$ Research Center for Eco-Environmental Sciences, Chinese Academy of Sciences, Beijing 100085, PR China
}

\section{A R T I C L E I N F O}

\section{Article history:}

Received 16 December 2009

Received in revised form 27 February 2010

Accepted 1 March 2010

Available online 7 March 2010

\section{Keywords:}

Polyelectrolyte multilayer membrane

Post-treatment

Hollow fiber

Pervaporation

Dynamic layer-by-layer process

\begin{abstract}
A B S T R A C T
The layer-by-layer ( $\mathrm{LbL}$ ) assembled polyelectrolyte multilayer has recently been recognized as a new class of promising membrane material for various separation uses. However, there is a lack of understanding about the influences of separation mixtures on the adsorbed polyelectrolytes. Therefore, clear understanding on it would be very important for the design and application of a type of new functional composite membrane. In this paper, the multilayer of weak polyelectrolytes polyethyleneimine and polyacrylic acid was constructed onto a hydrolyzed hollow fiber polyacrylonitrile support membrane under a negative pressure condition. The salt-, $\mathrm{pH}$ - and oxidant-responsive pervaporation behaviors of polyelectrolyte multilayer membranes were evaluated by post-treating with sodium chloride, hydrochloric acid, sodium hydroxide and sodium hypochlorite aqueous solutions, respectively. The pervaporation performances for separation of ethanol/water were compared before and after post-treatments. Scanning electron microscopy and atomic force microscopy confirmed the microtopographical changes of membrane surfaces. Optical microscopy was also used to real-time observe surface morphologies of polyelectrolyte multilayers deposited on the quartz substrates. Finally, the comparison of zeta potential values of inner surface before and after post-treatment also demonstrated the changes of surface electrical property.
\end{abstract}

(C) 2010 Elsevier B.V. All rights reserved.

\section{Introduction}

The layer-by-layer ( $\mathrm{LbL}$ ) method is an attractive technique for the fabrication of multilayer thin films with tailored structures and composition. It has been demonstrated that the LbL assembled multilayer is particularly suitable for separation membranes. Recent studies have contributed to the progress in developing polyelectrolyte multilayer membranes (PEMMs) for the use of pervaporation dehydration [1-6] and water desalination [7-9]. For the electrostatic LbL assembled membranes, a strong interest in using weak polyelectrolytes has recently been developed. For example, detailed investigations have been made on multilayer membrane of the weak polyelectrolytes polyethyleneimine (PEI) and polyacrylic acid (PAA) concerning their adsorption properties, morphologies and pervaporation behavior [10-14]. However, less work has been focused on the $\mathrm{pH}-$, salt- and oxidant-responsive behavior of PEMMs, especially for the influences of separation performance. It is well-known that the real separation medium is usually relatively complicated. There is a possibility that the presence of salt, acid, alkali and oxidant in the feed solution would affect the separation

\footnotetext{
* Corresponding authors. Fax: +86 1067392393.

E-mail addresses: zhanggj@bjut.edu.cn (G. Zhang), jshl@bjut.edu.cn (S. Ji).
}

capacity of PEMMs [15-17]. Even in the cases of membrane fouling and cleaning processes, the salt-, $\mathrm{pH}$ - and oxidant-tolerances are very important for the application of PEMMs. In fact, a few studies have made great efforts to investigate the effects of $\mathrm{pH}$ and salt on the building up and post-treatment of polyelectrolyte multilayers films [18-31]. For instance, Choi and Rubner [26] reported that the charge density of weak polyelectrolyte depends on changes of the local environment. The charge density of polycation is high at a low $\mathrm{pH}$ value and the ionization of polyanion is restrained, while the situation is reversed at a high $\mathrm{pH}$ condition. Similarly, the ionization of adsorbed polyelectrolyte can be modulated by changing the ionic strength. Xie and Granick [30] reported that even weak polyelectrolyte embedded at the bottom of 10-layer can be influenced by high ionic strength. However, all these studies limited to the polyelectrolyte multilayers on rigid substrates such as quartz and silica wafers. For the purpose of separation application, it is necessary to understand the salt-, $\mathrm{pH}$ - and oxidant-responsive behaviors of PEMMs on polymeric substrates.

Recently, we have prepared polyelectrolyte multilayers on polyacrylonitrile (PAN) hollow fiber support membrane [31,32]. Specifically, $1 \mathrm{~m}$ long pilot-scale module was successfully fabricated for pervaporation dehydration. This result suggested that the LbL assembled membrane has great potential for industrial application [31]. In this work, the pervaporation dehydration per- 
formances were compared before and after the post-treatments with hydrochloric acid, sodium hydroxide, sodium chloride and sodium hypochlorite, respectively. The microtopographical changes of the hollow fiber PEMMs were observed using a scanning electron microscopy (SEM) and an atomic force microscopy (AFM). Optical microscope pictures were taken on rigid quartz substrates in order to further explore the driven mechanisms. The zeta potential changes of hollow fiber inner surfaces were also characterized.

\section{Experimental}

\subsection{Materials}

The hollow fiber PAN ultrafiltration (UF) membrane was produced by dry/wet phase inversion method in our workshop [33]. The molecular weight cut-off of the fresh PAN UF membrane was about 60,000 Da. Quartz substrates used in this study were provided by Beijing Kinglass Quartz Co. Ltd. Branched poly(ethyleneimine) (PEI) with a molecular weight of $60,000 \mathrm{Da}$ was purchased from ACROS. The PAA powder with average molecular weight of 4,000,000 Da was obtained from Aldrich. Sodium chloride, sodium hydroxide, hydrochloric acid, sodium hypochlorite and ethanol were supplied by Beijing Chemical Factory. All the chemicals were of analytical grade and used without further purification.

\subsection{Post-treatments of polyelectrolyte multilayer membranes}

The preparation of PEMMs on a hydrolyzed PAN hollow fiber membrane was conducted by using a dynamic negative LbL technique, which has been reported in our previous studies [31,32]. After the stable state was reached, the LbL assembled membranes were post-treated with different solutions for $1 \mathrm{~h}$ by dynamically recycling respective sodium chloride, hydrochloric acid, sodium hydroxide and sodium hypochlorite aqueous solutions. The recycling velocity was maintained at $0.3 \mathrm{~m} / \mathrm{s}$ while the temperature was kept at $25^{\circ} \mathrm{C}$. The membranes were then rinsed with deionized water for approximately $20 \mathrm{~min}$ and dried in an oven at $50^{\circ} \mathrm{C}$. The post-treated membranes were subsequently evaluated for pervaporation of $95 \mathrm{wt} . \%$ ethanol/water mixtures. The above experiments were repeated for a determined cycles.

\subsection{Preparation of polyelectrolyte multilayer on quartz slides}

In this study, the polyelectrolyte multilayers were also assembled on quartz substrates for optical microscope observation. Before assembly, the quartz slides were pre-treated with a boiling piranha solution ( $70 \% \mathrm{H}_{2} \mathrm{SO}_{4} / 30 \% \mathrm{H}_{2} \mathrm{O}_{2}$ : caution: piranha is a strong oxidizer and should not be stored in closed containers) for $5 \mathrm{~h}$ and rinsed in de-ionized water. Then the quartz slides were alternatively immersed into PEI and PAA aqueous solutions for $30 \mathrm{~min}$ and followed by rinsing with de-ionized water before the next layer assembly. Polyelectrolyte multilayers were dried with a nitrogen stream.

\subsection{Pervaporation experiments}

The pervaporation dehydration performances of $95 \mathrm{wt} . \%$ ethanol/water mixture were compared before and after respective post-treatments. A specially designed laboratory fabricated pervaporation system was used to evaluate the hollow fiber membrane modules. In this system, the lumen side of the hollow fibers was used as the feed side, while the shell side was chosen as the permeate side. Fluxes were assessed by measuring the weight of liquid collected in the cold traps under the steady-state conditions. The composition of the collected permeate was evaluated by gas chromatography (GC-14C, SHIMADZU). The downstream pressure was

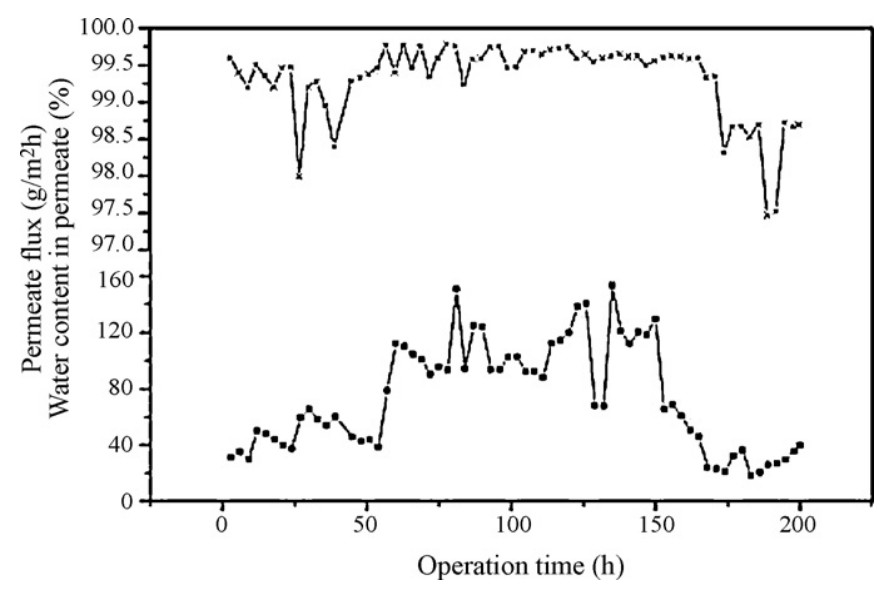

Fig. 1. Operation stability of hollow fiber PEMMs for pervaporation separation on the ethanol/water mixture (hydrolysis conditions for PAN support membrane: hydrolysis temperature $65^{\circ} \mathrm{C}$, hydrolysis time $15 \mathrm{~min}$; preparative conditions: 3.5 bilayers, $30 \mathrm{~min}$ filtration time, $0.05 \mathrm{wt}$.\% PAA aqueous solution, $0.25 \mathrm{wt}$ \% PEI aqueous solution, dynamic negative pressure $-0.09 \mathrm{MPa}, 25^{\circ} \mathrm{C}$; pervaporation conditions: feed temperature $50^{\circ} \mathrm{C}$, permeate pressure $100 \mathrm{~Pa}$, EtOH content in feed solution $95 \mathrm{wt} . \%)$.

about $100 \mathrm{~Pa}$. The separation factor was calculated using the quotient of the weight ratio of water and alcohol in the permeate, $Y_{\mathrm{HOH}} / Y_{\mathrm{ROH}}$, and in the feed, $X_{\mathrm{HOH}} / X_{\mathrm{ROH}}$ :

$\alpha=\frac{Y_{\mathrm{HOH}} / Y_{\mathrm{ROH}}}{X_{\mathrm{HOH}} / X_{\mathrm{ROH}}}$

\subsection{Characterization}

The inner surface morphologies of the PAN-based hollow fiber polyelectrolyte multilayer membranes ( 3.5 bilayers) were observed with SEM (Hitachi, Model S-4300) and AFM (Digital Instrument, DI 5000). All membrane samples were dried under the vacuum. An optical microscopy (Olympus BX51) was used to characterize the morphology changes of multilayers assembled on quartz substrates (20.5 bilayers). Zeta potentials were determined using an Electrokinetic analyzer (Anton Paar, SurPASS). In the process for measuring the zeta potential, the $\mathrm{KCl}$ solution concentration was maintained at $1.0 \mathrm{~mol} / \mathrm{L}$, while the operation pressure was $0.03 \mathrm{MPa}$. Attenuated total reflectance FTIR spectra were obtained using a Vertex-70 spectrophotometer (Bruker, Germany). Measurements were carried out at room temperature.

\section{Results and discussion}

\subsection{Effects of different post-treatment condition}

The stability of PEMMs is a major concern for its separation application. In this study, the LbL assembled hollow fiber PEMMs was firstly operated over $200 \mathrm{~h}$ for pervaporation separation of $95 \mathrm{wt}$ \% ethanol/water mixture. The experimental results in Fig. 1 clearly indicated that although the separation selectivity varied a little over the operation time, the water content in permeate always maintain above $97.5 \mathrm{wt} . \%$. This suggested the relatively good long-term stability of the PEMMs in the separation of binary solvent/water system. Of note, the variations of permeate flux were also observed in the experiments. This is because that the pervaporation is a very exact process in the laboratory. Especially for such a small membrane module, only a small amount of the permeate was collected in each cycle, therefore, any minute operating and weighting errors would lead to the fluctuation. However, the accurate selectivity data 

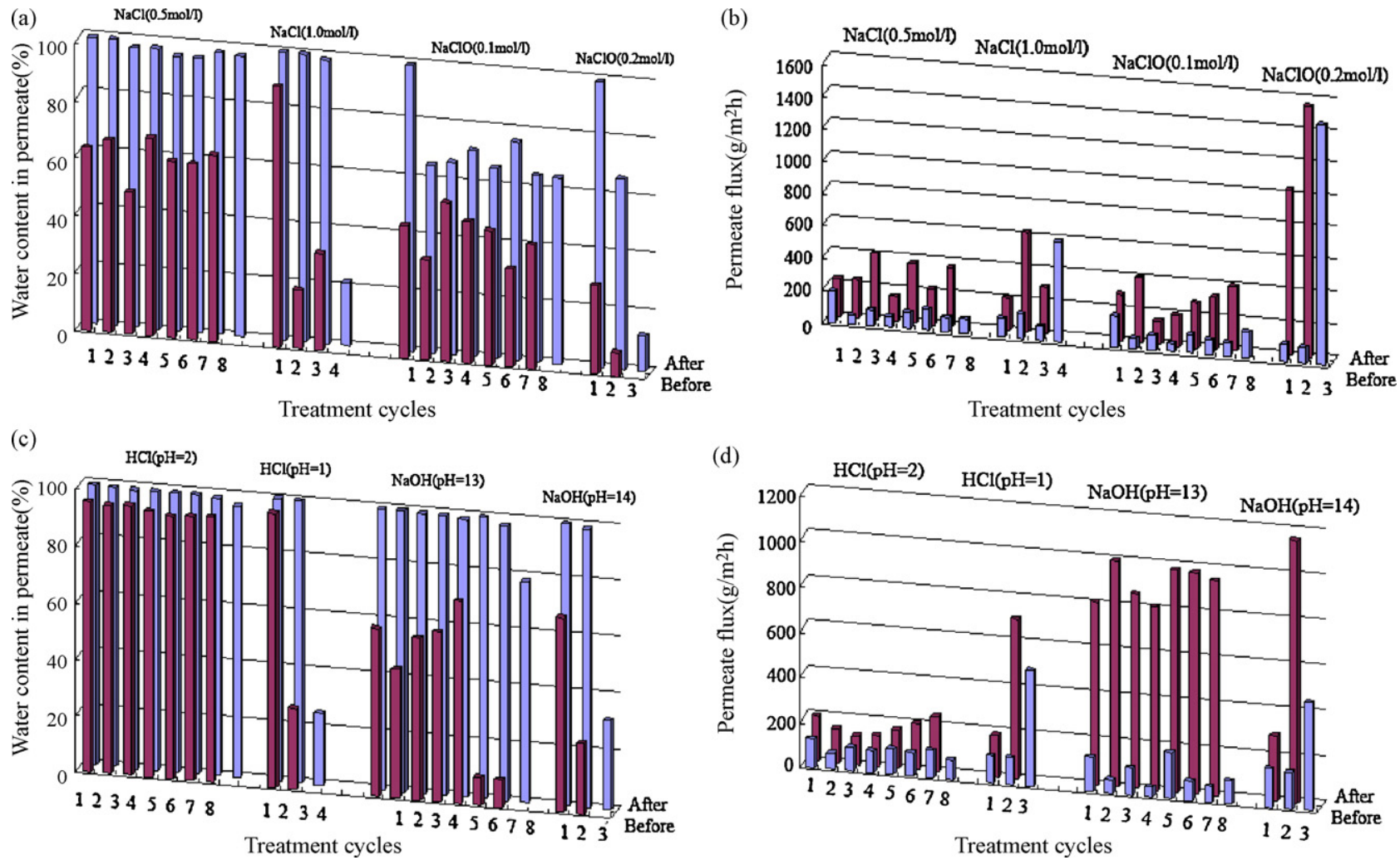

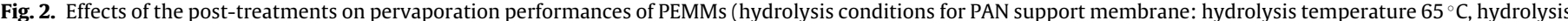

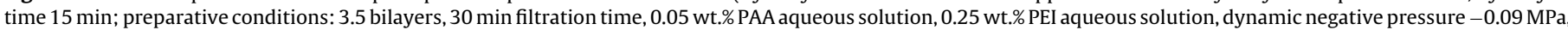
$25^{\circ} \mathrm{C}$; pervaporation conditions: feed temperature $50{ }^{\circ} \mathrm{C}$, permeate pressure $100 \mathrm{~Pa}$, EtOH content in feed solution $95 \mathrm{wt} . \%$ ).

could be achieved by using GC determination. Subsequently, the influences of salt, $\mathrm{pH}$ and oxidant post-treatments on the performance of PEMMs were evaluated within several consecutive cycles.
Fig. 2 shows the variations of PEMMs with the four different post-treatments. It was noted that the performance of PEMMs was sensitive to the salt solution. For example, the water content in permeate decreased from $99.2 \%$ to $63.4 \%$ after the first cycle treat-

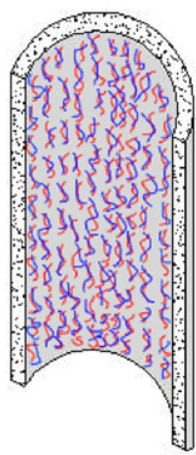

Treated with $\mathrm{NaCl}$ solution

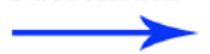

Polycation(PEI)

Polyanion(PAA)
Rinsed with de-ionized water and dried
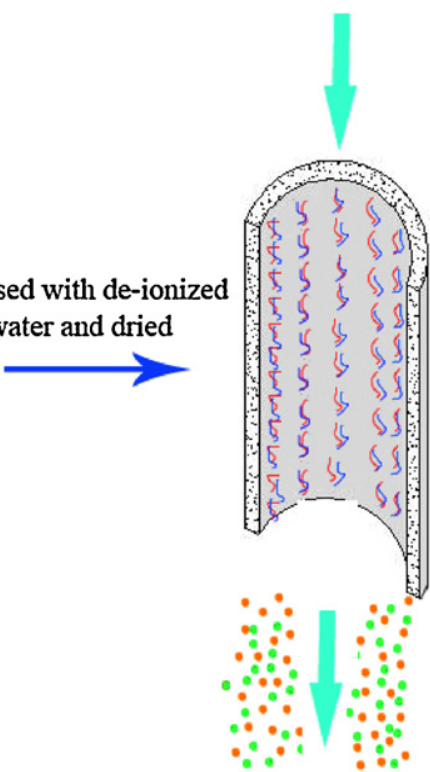

- $\mathrm{Na}^{+}$

- $\mathrm{Cl}^{-}$

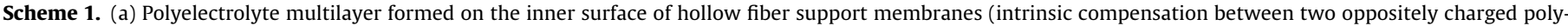
electrolyte); (b) multilayer swelling and partially extrinsic compensation by salt ions; (c) rearrangement of polyelectrolyte complexes after rinsing with pure water. 
ment with $0.5 \mathrm{~mol} / \mathrm{L} \mathrm{NaCl}$ solution. The reason is that the increasing ionic strength of the system could lead to the salt swelling and partially decomposition of the multilayer films $[16,19,20]$. The changes of multilayer film with the salt post-treatment are presented in Scheme 1. As shown in Scheme 1, before $\mathrm{NaCl}$ post-treatment, the polycation charges are compensated by polyanion charges (intrinsic compensation). During $\mathrm{NaCl}$ post-treatment process, the external salt solution would lead to multilayer swelling to certain extent. The counter ions including $\mathrm{Na}^{+}$and $\mathrm{Cl}^{-}$can enter into the interior of multilayer and thereby compensate the polymer charges, and the compensation is extrinsic. Therefore, the polyelectrolyte chains could become tortuous and in turn rearrange at the surface of support membrane. This might result in looser structure and lower selectivity of the membranes. When the swelling is sufficient, the multilayer will partially decompose and go back into the external solution. The similar phenomena were also observed in the case of multilayer films on the rigid substrates by other research groups [18-20,22]. However, it was also noted that pervaporation selectivity could mostly recover after the multilayer being rinsed and dried. For example, the water content in permeate recovered from $63.4 \%$ to $96.9 \%$ in the second cycle. This is because the counter ions $\left(\mathrm{Na}^{+}\right.$and $\left.\mathrm{Cl}^{-}\right)$could be washed away from the interior of multilayer during the rinsing step inbetween operation cycles (Scheme 1). The compensation of the polymer charges would then strongly depend on the charge balance between polycation and polyanion. Consequently, after drying step, the polyelectrolyte chain will re-settle and move towards the previous status. Of note, although a part of outer multilayer decomposed during the $\mathrm{NaCl}$ post-treatment, the multilayer itself has a cross-sectional thickness. Therefore, the membrane performance could mostly recover. This also suggested that the salt swelling might be recognized as the main reason contributed to the decline of selectivity in the case of a relatively low salt concentration. It is also noted that the salt concentration has great influence on the pervaporation performance of the multilayer membrane. For example, in the process of post-treatment with $1.0 \mathrm{~mol} / \mathrm{L} \mathrm{NaCl}$, the membrane selectivity could not recover after 3 cycles. This fact suggested that the multilayer could be completely destructed by consecutive post-treatment with the high concentration of salt (Fig. 2(a)).

It is well-known that the ionization degree of weak polyelectrolyte is strongly dependent on the local $\mathrm{pH}$ conditions, which could influence the separation performance of PEMMs $[34,35]$. As shown in Fig. 2(c) and (d), the permeate flux increased, while the water content in the permeate declined from about $99.0 \%$ to $95.0 \%$ after post-treating with $\mathrm{HCl}$ solution $(\mathrm{pH}=2)$. Similar to the results observed in the previous $\mathrm{NaCl}$-involved experiment, pervaporation performance could mostly recover after rinsing and drying steps in the next cycle. When treating with $\mathrm{NaOH}$ solution $(\mathrm{pH}=13)$, similar phenomenon was observed. Obviously, although polyelectrolytes were deposited on the support membrane, the effects of $\mathrm{pH}$ conditions on charge density were notable. Dubas and Schlenoff had reported that PAA exhibits gradual dissociation over a range of solution $\mathrm{pH}$ values. About 50\% PAA could be protonated at the $\mathrm{pH}$ of 5.0 and completely ionized over 9.0 [18]. The $\mathrm{pH}$ variation could destroy the balance of electrostatic force and changed the thickness of polyeletrolyte multilayers [23]. Furthermore, similar to the salt case, the small counter ion could also compensate the polymer charges, which would cause the swelling and in turn looser structure of multilayer. These might be the possible reason that caused the variation of membrane performance. Moreover, it was noted that the membrane performance could not be recovered after two cycles treatments under the extreme $\mathrm{pH}$ conditions, such as $\mathrm{pH}=1$ and $\mathrm{pH}=14$. These results also suggested that the polyelectrolyte complexes could almost be dissociated under the extreme $\mathrm{pH}$ conditions.

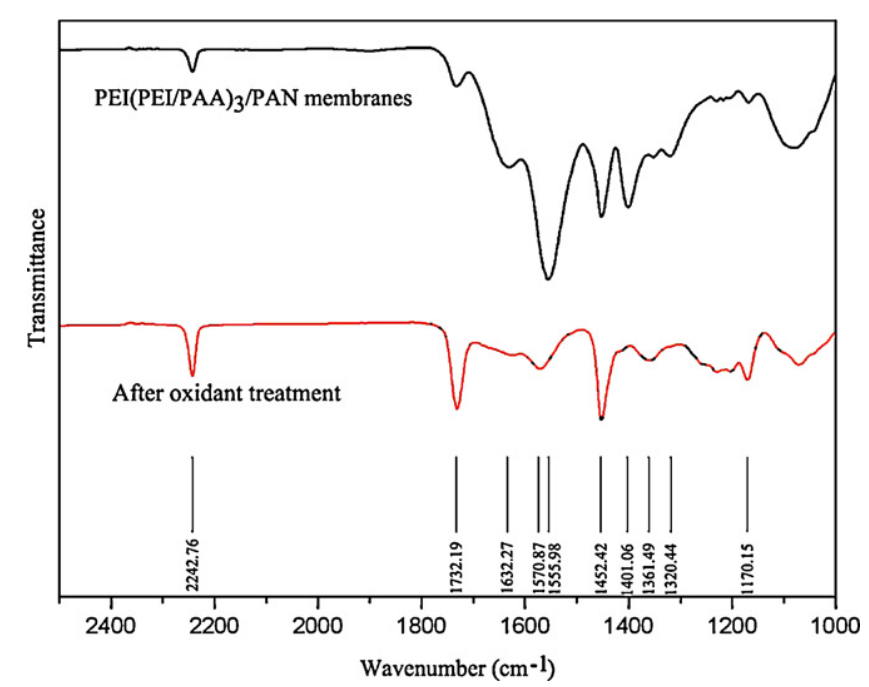

Fig. 3. FTIR spectra of PAN-based PEMMs before and after post-treatment with $\mathrm{NaClO}$ solution (hydrolysis conditions for PAN support membrane: hydrolysis temperature $65^{\circ} \mathrm{C}$, hydrolysis time $15 \mathrm{~min}$; preparative conditions: 3.5 bilayers, $30 \mathrm{~min}$ filtration time, 0.05 wt.\% PAA aqueous solution, 0.25 wt.\% PEI aqueous solution, dynamic negative pressure $-0.09 \mathrm{MPa}, 25^{\circ} \mathrm{C}$; pervaporation conditions: feed temperature $50{ }^{\circ} \mathrm{C}$, permeate pressure $100 \mathrm{~Pa}$, EtOH content in feed solution $95 \mathrm{wt} . \%$; treatment time $1 \mathrm{~h}$, concentration of $\mathrm{NaClO}$ solution $0.1 \mathrm{~mol} / \mathrm{L}$ ).

Sodium hypochlorite is widely used for membrane cleaning. In this study, the oxidant tolerance of PEMMs was tested. Water content in permeate decreased approximately from $99.0 \%$ to $40.0-50.0 \%$ after post-treating with $0.1 \mathrm{~mol} / \mathrm{L}$ sodium hypochlorite solution (Fig. 2). Meanwhile, the increase in the concentration of sodium hypochlorite solution could also speed up the destruction of multilayer membrane. For example, the water content in permeate could decreased to below $10.0 \%$ after only two cycles post-treatment of $0.2 \mathrm{~mol} / \mathrm{L} \mathrm{NaClO}$ solution. Different from $\mathrm{pH}-$ and salt-responsive behaviors, the oxidant post-treatment might destroy the polyelectrolyte chain and in turn lead to the irreversible decline of selectivity. To further verify the changes of multilayer structure, infrared spectra of PEMMs before and after treating with $\mathrm{NaClO}$ solution are compared in Fig. 3. After post-treating with $\mathrm{NaClO}$ solution, the characteristic peaks at 1555 and $1401 \mathrm{~cm}^{-1}$, which were due to the $-\mathrm{COO}^{-}$group $[24,36,37]$, sharply weakened. As a comparison, the peak at $1732 \mathrm{~cm}^{-1}$ attributed to the $-\mathrm{C}=\mathrm{O}$ group became much stronger $[11,38]$. These results further confirmed that most of $-\mathrm{C}=\mathrm{O}$ groups of the support membrane was exposed due to the destruction of outer multilayer. Moreover, the intensities of -CN group of PAN support membrane (at 2242 and $1452 \mathrm{~cm}^{-1}$ ) increased, which suggested that the polyelectrolyte multilayer assembled on support membrane was destroyed and partially lost after oxidation.

\subsection{FESEM, AFM and optical microscopy experiments}

In order to understand the microtopographical changes on membrane surfaces, SEM and AFM tests were preformed. Fig. 4(a) indicates that a dense and defect-free selective layer is formed on the support membrane after depositing 3.5 polyelectrolyte bilayers. The images of membranes treated with $\mathrm{NaCl}, \mathrm{HCl}, \mathrm{NaOH}$ and $\mathrm{NaClO}$ solutions under different conditions are shown in Fig. 4(b)-(m), respectively. Comparing Fig. 4(a)-(c), it could be obviously observed that the polyelectrolyte multilayers on the top surface become looser due to the swelling of salt solution. After the rinsing step, the polyelectrolyte chain could stretch and partially recover the surface. Under the extremely high salt concentration conditions, a few of big defects were formed on the 
(a)

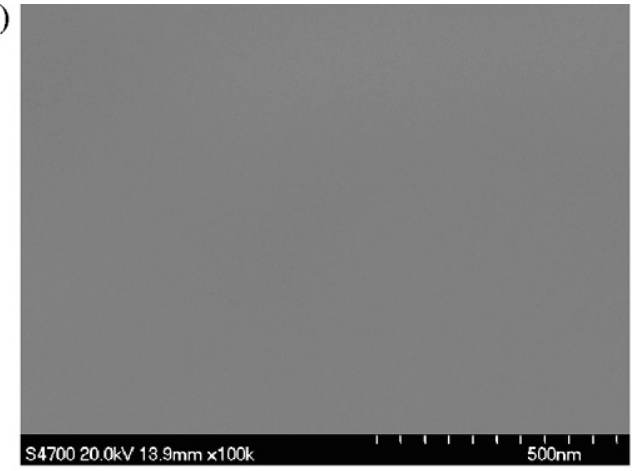

(c)

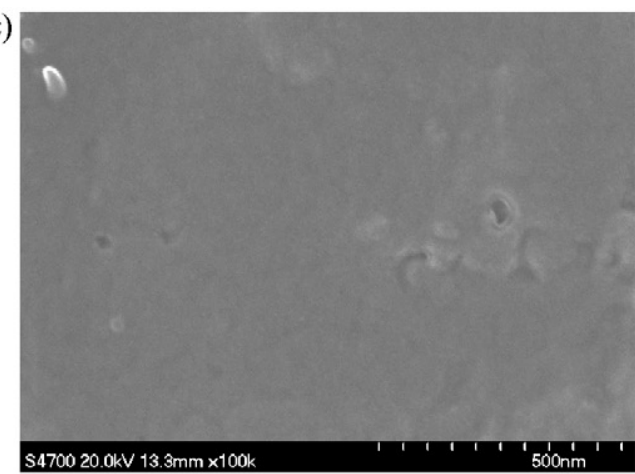

(e)

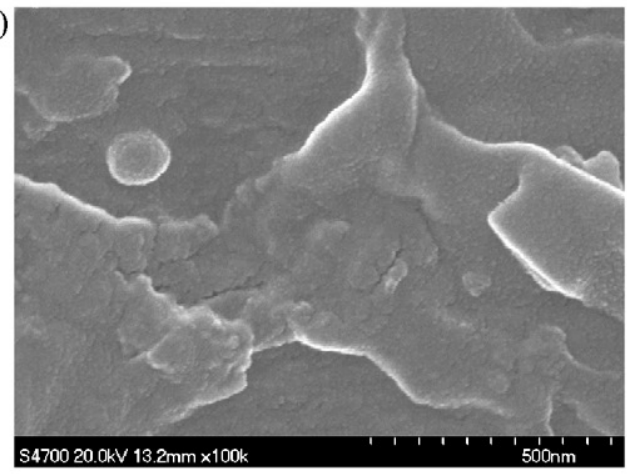

(b)

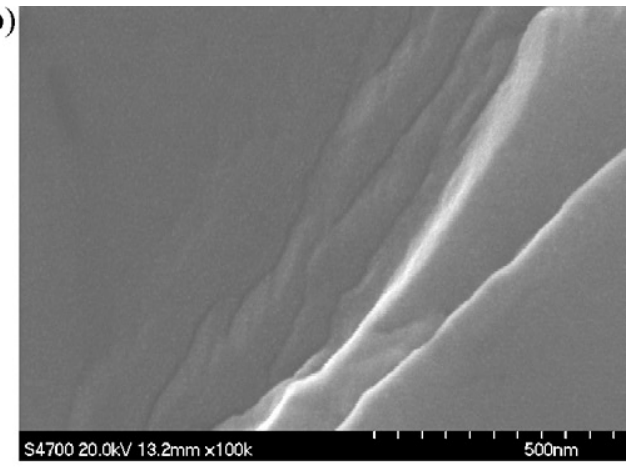

(d)

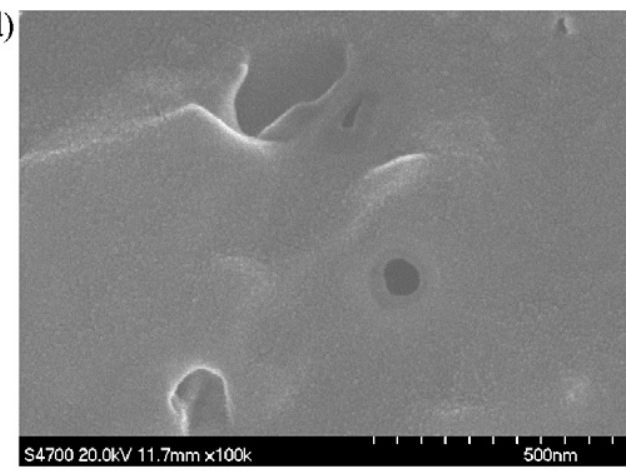

(f)

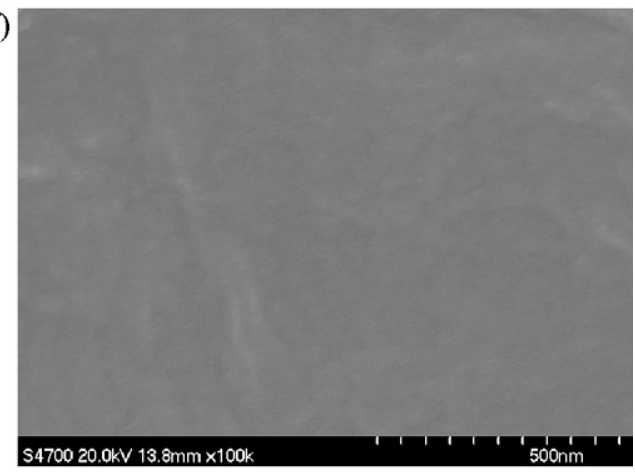

(g)

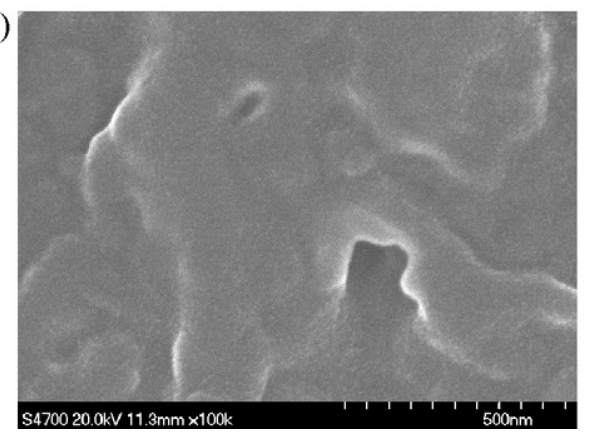

Fig. 4. SEM pictures of the hollow fiber PEMMs post-treated by different solutions. (a) SEM of inner surface before post-treatment (100,000 $\times$ ). (b) SEM of inner surface after post-treatment with $0.5 \mathrm{~mol} / \mathrm{L} \mathrm{NaCl}$ solution $(100,000 \times)$. (c) SEM of inner surface after post-treatment with $0.5 \mathrm{~mol} / \mathrm{L} \mathrm{NaCl}$ solution and rinsing with pure water (100,000 $\times$ ). (d) SEM of inner surface after post-treatment with $1.0 \mathrm{~mol} / \mathrm{L} \mathrm{NaCl}$ solution $(3 \mathrm{cycles})(100,000 \times$ ). (e) $\mathrm{SEM}$ of inner surface after post-treatment with $\mathrm{HCl}$ solution ( $\mathrm{pH}=2$ ) $(100,000 \times)$. (f) SEM of inner surface after post-treatment with $\mathrm{HCl}$ solution $(\mathrm{pH}=2)$ and rinsing with pure water $(100,000 \times)$. $(\mathrm{g})$ SEM of inner surface after post-treatment with $\mathrm{HCl}$ solution $(\mathrm{pH}=1,3$ cycles $)(100,000 \times)$. (h) SEM of inner surface after post-treatment with $\mathrm{NaOH}$ solution $(\mathrm{pH}=13)(100,000 \times)$. (i) SEM of inner surface after post-treatment with $\mathrm{NaOH}$ solution $(\mathrm{pH}=13)$ and rinsing with pure water $(100,000 \times)$. (j) SEM of inner surface after post-treatment with $\mathrm{NaOH}$ solution $(\mathrm{pH}=14,3 \mathrm{cycles})(100,000 \times)$. $(\mathrm{k}) \mathrm{SEM}$ of inner surface after post-treatment with $\mathrm{NaClO}$ solution $(0.1 \mathrm{~mol} / \mathrm{L})(100,000 \times)$. (l) SEM of inner surface after post-treatment with $\mathrm{NaClO}$ solution $(0.1 \mathrm{~mol} / \mathrm{L})$ and rinsing with pure water $(100,000 \times)$. (m) SEM of inner surface after post-treatment with $\mathrm{NaClO}$ solution $(0.2 \mathrm{~mol} / \mathrm{L}, 3$ cycles) $(100,000 \times)$ (hydrolysis conditions for PAN support membrane: hydrolysis temperature $65^{\circ} \mathrm{C}$, hydrolysis time $15 \mathrm{~min}$; preparative conditions: 3.5 bilayers, 30 min filtration time, 0.05 wt.\% PAA aqueous solution, 0.25 wt.\% PE aqueous solution, dynamic negative pressure $-0.09 \mathrm{MPa}, 25^{\circ} \mathrm{C}$; pervaporation conditions: feed temperature $50^{\circ} \mathrm{C}$, permeate pressure $100 \mathrm{~Pa}$, EtOH content in feed solution 95 wt.\%). 
(h)

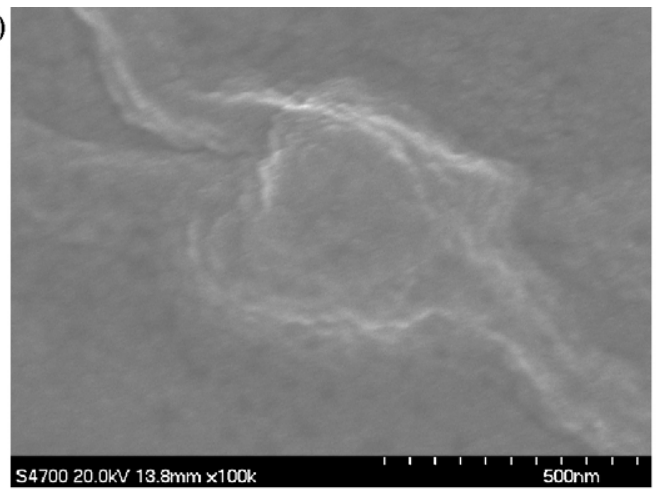

(i)

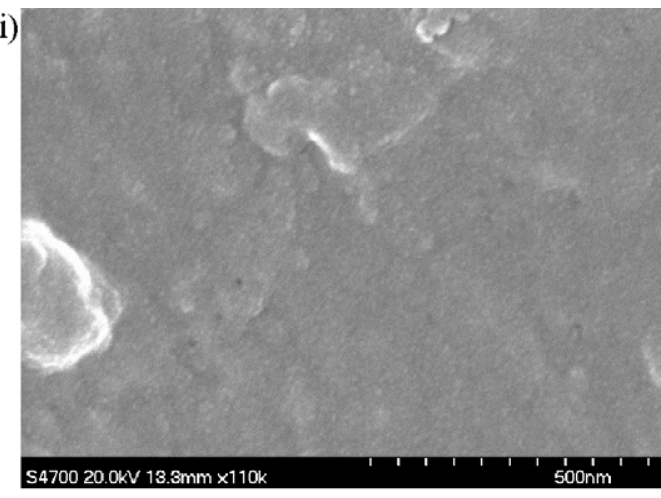

(j)

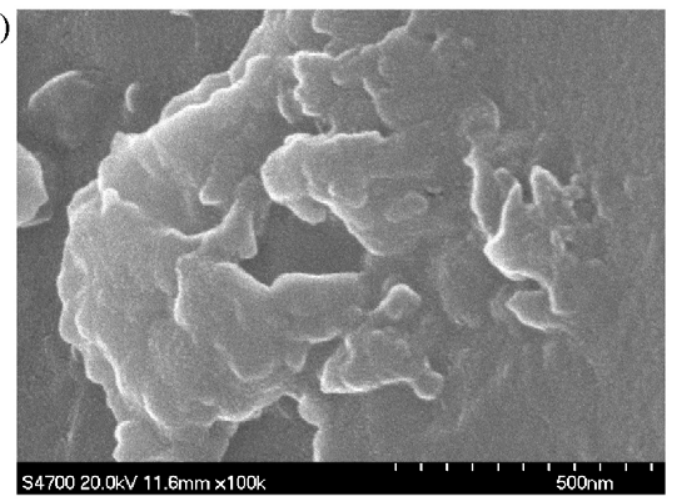

(k)

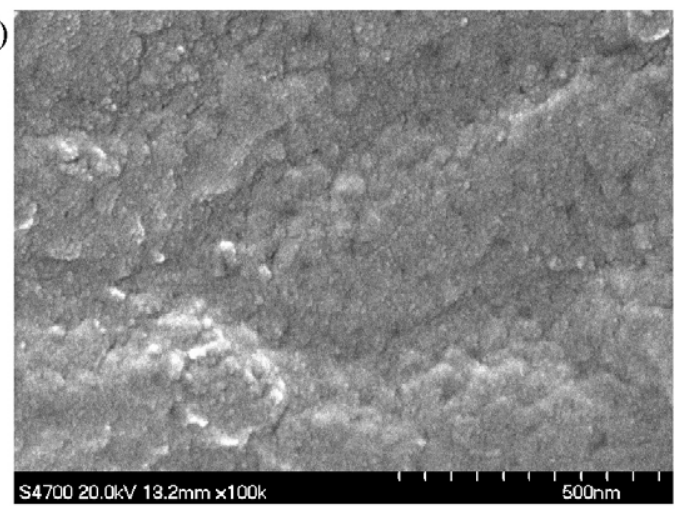

(1)

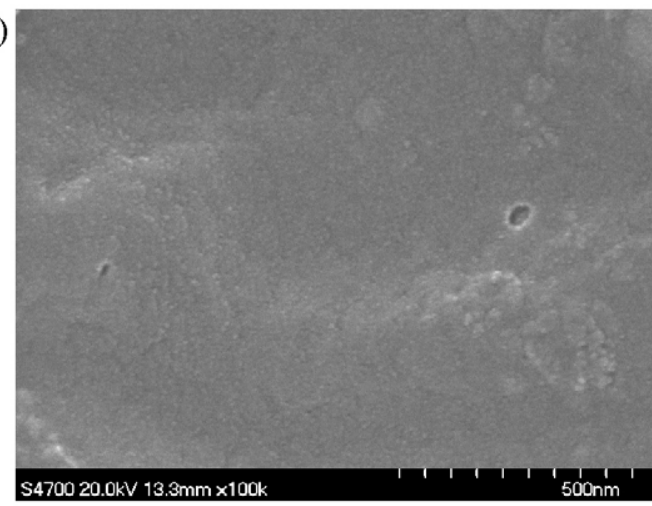

(m)

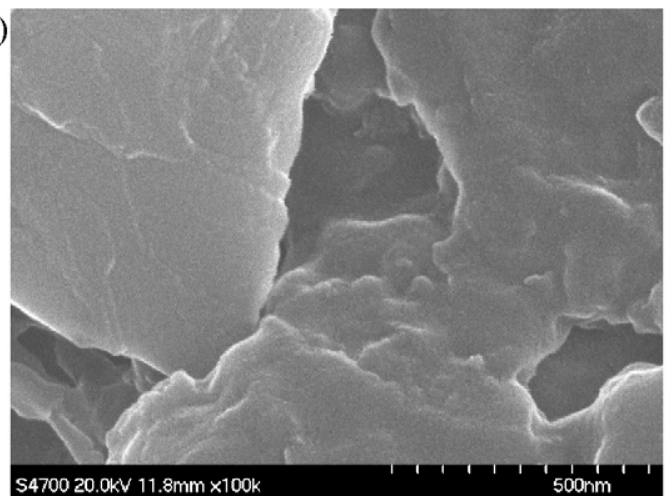

Fig. 4. (Continued)

swelling surface (Fig. 4(d)). In comparison with Fig. 4(a), (e) and (h), it is found that the membrane also become much looser in the case of acid and alkaline treatment. The polyelectrolyte complex aggregated due to the change of charge balance between oppositely charged polyelectrolytes and the swelling of counter ions. Similarly, after rinsing with de-ionized water, to some extent, the membrane surface recovered. Under the extreme $\mathrm{pH}$ conditions, the polyelectrolyte multilayer swelled and aggregated much more severely, and consequently caused the irreversible deterioration of membrane performance. When the post-treatment is carried out using $\mathrm{NaClO}$ solution (Fig. $4(\mathrm{k})$ ), a large number of the defects can also be clearly observed. The structures of polyelectrolyte multi- 

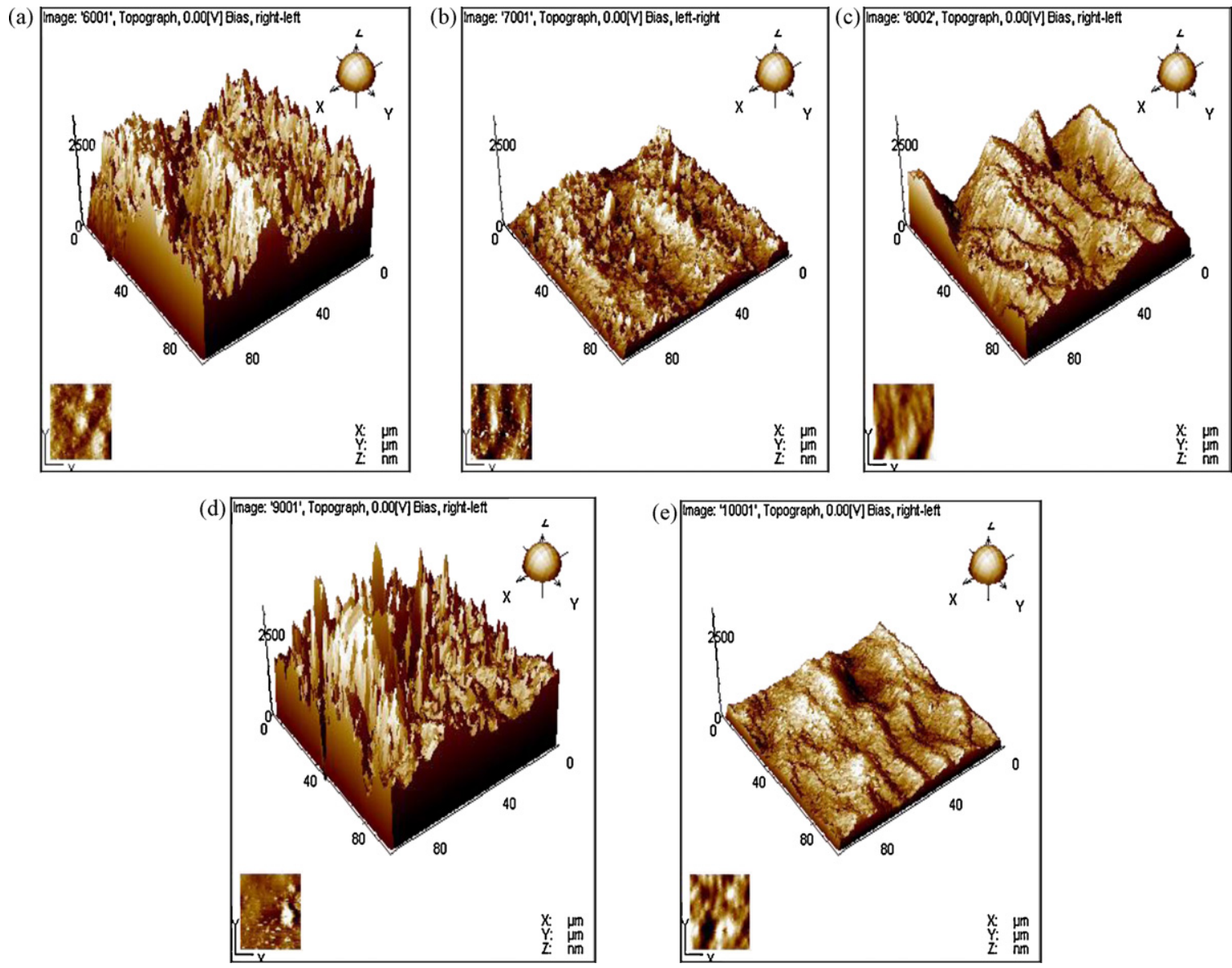

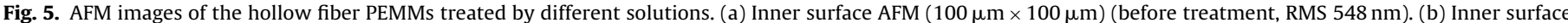

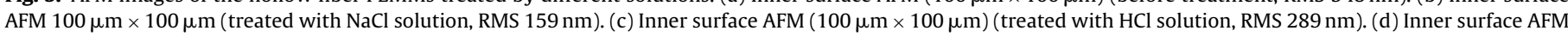

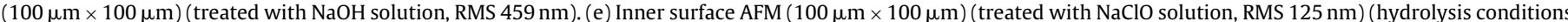

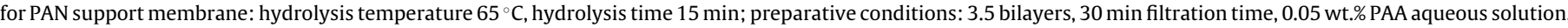

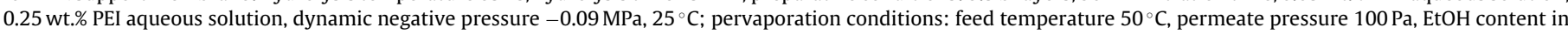
feed solution $95 \mathrm{wt} . \%$ ).

layer can be strongly destructed (Fig. 4(m)). In conclusion, these SEM images confirmed that the post-treatments with the salt, acid, alkali and oxidant can increase the opportunity for the formation of loose structure and big defects on membrane surfaces, and in turn results in the selectivity decline.

The top surface morphologies of the PEMMs are also observed by AFM. The three-dimensional AFM images of support membranes before and after post-treatment are presented in Fig. 5. The values of the root mean square roughness (RMS) are obtained based on $100.0 \mu \mathrm{m} \times 100.0 \mu \mathrm{m}$ scan area. It was noted that the top surfaces become smoother after the post-treatments with different solutions. For example, the RMS of the PEI/PAA multilayer membranes decreased from 548 to $159,289,459,125 \mathrm{~nm}$ after being treated with the $\mathrm{NaCl}, \mathrm{HCl}, \mathrm{NaOH}$ and $\mathrm{NaClO}$ solutions for $1 \mathrm{~h}$, respectively. The decrease of membrane surface roughness might be attributed to the chain rearrangement and surface loss of the polyelectrolytes
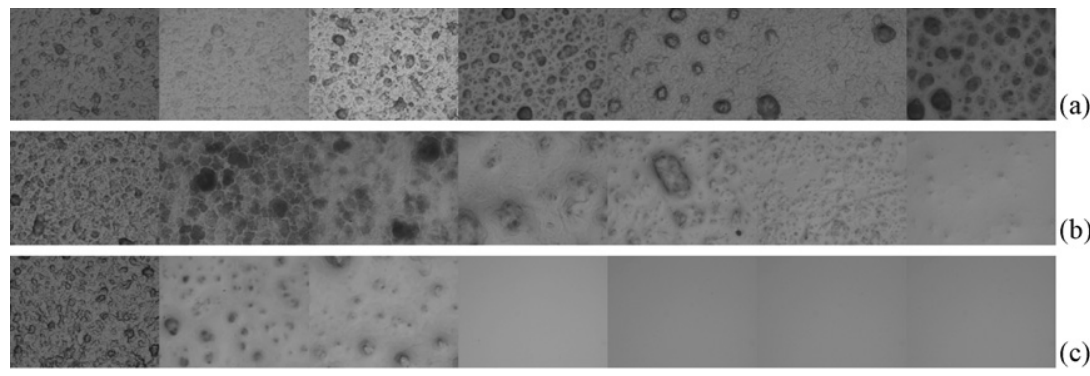

b)

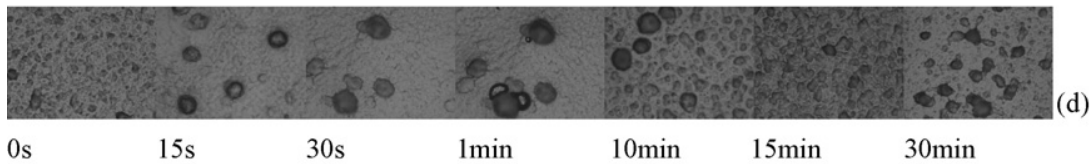

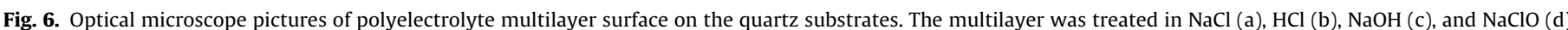
solution at $0-30 \mathrm{~min}(100 \times)$ (preparative conditions: 20.5 bilayers, $30 \mathrm{~min}$ assemble time, 0.05 wt.\% PAA aqueous solution, and 0.25 wt.\% PEI aqueous solution). 
Table 1

Changes of zeta potential before and after post-treatments.

\begin{tabular}{|c|c|c|}
\hline Post-treatment solutions & Zeta potential before post-treatments (mV) & Zeta potential after post-treatments (mV) \\
\hline $\mathrm{HCl}(\mathrm{pH}=2)$ & +6.572 & +12.480 \\
\hline $\mathrm{NaOH}(\mathrm{pH}=13)$ & +5.848 & +3.436 \\
\hline $\mathrm{NaCl}(0.5 \mathrm{~mol} / \mathrm{L})$ & +5.838 & +2.528 \\
\hline $\mathrm{NaClO}(0.1 \mathrm{~mol} / \mathrm{L})$ & +6.896 & -7.911 \\
\hline
\end{tabular}

due to the salt-, pH- and oxidant-responsive behaviors.

In order to avoid the influence of polymeric porous support membranes, it is considered that the multilayer films on rigid quartz substrates can better show the changes of pure polyelectrolyte complex under different post-treatment conditions. Subsequently, the multilayers are assembled on quartz slides for the observation of optical microscopy. The reference information for the changes of pure polyelectrolyte multilayers is then obtained. The optical morphologies of PEI/PAA assembled on the quartz substrates with different post-treatment time are shown in Fig. 6. When exposing into $\mathrm{NaCl}$ solution (Fig. 6(a)), a number of micro-scale pores appear in the multilayer since the mobility and stretching of polyelectrolyte chain are limited at a high ionic strength condition. Moreover, the pores become much larger with the prolonged exposure time. This is due to the sufficient salt swelling and the corresponding aggregation of polyelectrolyte complexes under the salt environment. Fig. 6(b) clearly shows that the pores in the multilayer film firstly become larger and then disappear when the exposure time increased in the case of $\mathrm{HCl}$ post-treatment. The reason is that the film peeled off from the substrate after $30 \mathrm{~min} \mathrm{HCl}$ post-treatment. Recently, similar optical microscopy experiments have been performed by Lutkenhaus et al. [24]. The micro-scale pores are also observed in the post-treatment of PEI/PAA multilayer film with acid solution. It can also be observed from Fig. 6(c) that the polyelectrolyte multilayers completely peel off after exposing into $\mathrm{NaOH}$ solution for $1 \mathrm{~min}$. This result is consistent with the above pervaporation, SEM and AFM results. Fig. 6(d) also indicates the formation of microscale pores during the post-treatment with $\mathrm{NaClO}$ solution. This further demonstrates the destruction of multilayer structure after oxidization. Different from the rigid quartz substrates, the polymeric substrates are relatively flexible, elastic and rough. Moreover, polymeric ultrafiltration membrane substrate has a great number of nano-scale pores and a relatively wide pore diameter distribution. Therefore, to some extent, the interior of membrane pores can also be deposited with a few of polyelectrolyte multilayers. Even for the multilayers formed on the top surface, the forces between polymeric substrates and multilayers were relatively higher than those between rigid substrates and multilayer. The swelling and aggregation of polyelectrolyte complexes are consequently relatively weaker. Therefore, the formation of micro-scale pores and peeling off from substrates are not observed when the multilayers are assembled on polymeric porous support membranes.

\subsection{Zeta potential measurements}

Zeta potential is commonly employed to characterize the electrical properties of the polyelectrolyte multilayer films. It is also used to monitor the stepwise growth of sequential polymer layers [39]. The zeta potential of inner surface as a function of the number of polyelectrolyte layers is shown in Fig. 7. The zeta potential changes between +7 and $-10 \mathrm{mV}$ when PEI and PAA form the outer layers, respectively. The reversal change in the sign of the zeta potential indicates the stepwise growth of PEI and PAA on the hollow fiber substrates.

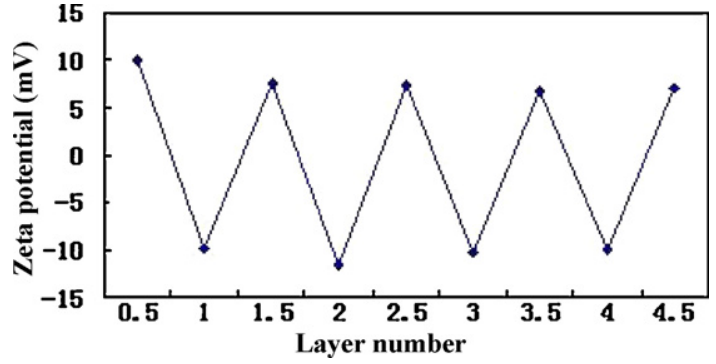

Fig. 7. Zeta potential changes with layer numbers (hydrolysis conditions for PAN support membrane: hydrolysis temperature: $65^{\circ} \mathrm{C}$, hydrolysis time $15 \mathrm{~min}$; preparative conditions: 4.5 bilayers, 30 min filtration time, 0.05 wt.\% PAA aqueous solution, 0.25 wt.\% PEI aqueous solution).

In this study, zeta potential is also used to characterize the change of surface properties. As shown in Table 1, zeta potential of inner surface increases from +6.572 to $+12.480 \mathrm{mV}$ after treating with $\mathrm{HCl}$ solution. That is because the PEI outer layer has positive charge in aqueous solutions. Treatment with $\mathrm{HCl}$ solution can result in the sufficient protonization of PEI and in turn the increase of zeta potential. Contrarily, the protonization of PEI can be efficiently restrained in alkaline solutions. Therefore, the zeta potential decreases from +5.848 to $+3.436 \mathrm{mV}$. It is also noted that zeta potential varies from +5.838 to $+2.528 \mathrm{mV}$ after the salt post-treatment. This is due to the reduction of ionized PEI at a relatively high ionic strength environment. Interestingly, the post-treatment with $\mathrm{NaClO}$ oxidant lead to the reversal charges. Zeta potential changed from +6.896 to $-7.911 \mathrm{mV}$. This result also suggests that the oxidization can destroy the PEI outer layer, which causes the exposure of prior negative PAA layer.

\section{Conclusions}

In this study, the salt-, $\mathrm{pH}$ - and oxidant-responsive behaviors of LbL assembled hollow fiber weak polyelectrolyte multilayer membranes are investigated. Although the hollow fiber PEMMs exhibits a relatively good solvent tolerance capacity, the pervaporation selectivity of ethanol/water mixture declines after post-treating with $\mathrm{NaCl}, \mathrm{HCl}, \mathrm{NaOH}$ and $\mathrm{NaClO}$ solutions, respectively. It is demonstrated that the post-treatments of the weak PEMMs with salt, acid, and alkali offer much opportunity for the formation of loose structure and big defects on multilayers due to the polyelectrolyte chain shape change. Under the high salt concentrations and extreme $\mathrm{pH}$ values, the deterioration of membrane performance is much more critical and irreversible. Particularly, the strong oxidant can destroy the outer polyelectrolyte layer and in turn result in the decline of selectivity. This suggests that the type of separation mixtures has a great influence on the stability of PEMMs. Therefore, to expand the application fields of PEMMs, it is necessary to seek for effective approaches to improve the stability of PEMMs in future study. In our opinion, thermal, chemical or photochemical covalent crosslinking could potentially be good options to achieve high stable PEMMs. 


\section{Acknowledgements}

This work was supported by the National Basic Research Program of China (no. 2009CB623404), the National Natural Science Foundation of China (no. 20806001), the Program for Beijing Excellent Talents (2009D005015000013), the Natural Science Foundation of Beijing (no. 8071001), Beijing Municipal Science and Technology Planning Project (no. Z08000603170801), and Funding Project for Academic Human Resources Development in Institutions of Higher Learning Under the Jurisdiction of Beijing Municipality (no. PHR201008018). The authors extended acknowledgements to Ms. Zhijuan Ji for helping us to correct the written English.

\section{References}

[1] L. Krasemann, A. Toutianoush, B. Tieke, Self-assembled polyelectrolyte multilayer membranes with highly improved pervaporation separation of ethanol/water mixtures, J. Membr. Sci. 181 (2001) 221-228.

[2] J.M. Haack, W. Lenk, D. Lehmann, K. Lunkwitz, Pervaporation separation of water/alcohol mixtures using composite membranes based on polyelectrolyte multilayer assemblies, J. Membr. Sci. 184 (2001) 233-243.

[3] P.D. Chapman, T. Oliveira, A.G. Livingston, K. Li, Membranes for the dehydration of solvents by pervaporation, J. Membr. Sci. 318 (2008) 5-37.

[4] J. Chen, Q. Liu, Y. Xiong, Q. Zhang, A. Zhu, Composite membranes prepared from glutaraldehyde cross-linked sulfonated cardo polyetherketone and its blends for the dehydration of acetic acid by pervaporation, J. Membr. Sci. 325 (2008) 184-191.

[5] Q. Zhao, J. Qian, Q. An, Z. Sun, Layer-by-layer self-assembly of polyelectrolyte complexes and their multilayer films for pervaporation dehydration of isopropanol, J. Membr. Sci. 346 (2010) 335-343.

[6] Q. Zhao, J. Qian, M. Zhu, Q. An, Facile fabrication of polyelectrolyte complex/carbon nanotube nanocomposites with improved mechanical properties and ultra-high separation performance, J. Mater. Chem. 19 (2009) 8732-8740.

[7] A. Toutianoush, W. Jin, $H$. Deligöz, B. Tieke, Polyelectrolyte multilayer membranes for desalination of aqueous salt solutions and seawater under reverse osmosis conditions, Appl. Surf. Sci. 246 (2005) 437-443.

[8] L. Ouyang, R. Malaisamy, M.L. Bruening, Multilayer polyelectrolyte films as nanofiltration membranes for separating monovalent and divalent cations, J. Membr. Sci. 310 (2008) 76-84.

[9] A. El-Hashani, A. Toutianoush, B. Tieke, Use of layer-by-layer assembled ultrathin membranes of dicopper-[18]azacrown- $\mathrm{N}_{6}$ complex and polyvinylsulfate for water desalination under nanofiltration conditions, J. Membr. Sci. 318 (2008) 65-70.

[10] G. Zhang, W. Gu, S. Ji, Z. Liu, Y. Peng, Z. Wang, Preparation of polyelectrolyte multilayer membranes by dynamic layer-by-layer process for pervaporation separation of alcohol/water mixtures, J. Membr. Sci. 280 (2006) 727-733.

[11] G. Zhang, H. Yan, S. Ji, Z. Liu, Self-assembly of polyelectrolyte multilayer pervaporation membranes by a dynamic layer-by-layer technique on a hydrolyzed polyacrylonitrile ultrafiltration membrane, J. Membr. Sci. 292 (2007) 1-8.

[12] Z. Zhu, X. Feng, A. Penlidis, Self-assembled nano-structured polyelectrolyte composite membranes for pervaporation, Mater. Sci. Eng. C 26 (2006) 1-8.

[13] S. Ji, G. Zhang, Z. Liu, Y. Peng, Z. Wang, Evaluations of polyelectrolyte multilayer membranes assembled by a dynamic layer-by-layer technique, Desalination 234 (2008) 300-306.

[14] P. Zhang, J. Qian, Q. An, X. Liu, Q. Zhao, H. Jin, Surface morphology and pervaporation performance of electric field enhanced multilayer membranes, J. Membr. Sci. 328 (2009) 141-147.

[15] M. Kolasińska, P. Warszyński, The effect of nature of polyions and treatment after deposition on wetting characteristics of polyelectrolyte multilayers, Appl. Surf. Sci. 252 (2005) 759-765.
[16] A. Escoda, P. Fievet, S. Lakard, A. Szymczyk, S. Déon, Influence of salts on the rejection of polyethyleneglycol by an NF organic membrane: pore swelling and salting-out effects, J. Membr. Sci. 347 (2010) 174-182.

[17] T. Yoshizawa, Y. Shin-ya, K. Hong, T. Kajiuchi, pH- and temperature-sensitive permeation through polyelectrolyte complex films composed of chitosan and polyalkyleneoxide-maleic acid copolymer, J. Membr. Sci. 241 (2004) 347-354.

[18] S.T. Dubas, J.B. Schlenoff, Polyelectrolyte multilayers containing a weak polyacid: construction and deconstruction, Macromolecules 34 (2001) 3736-3740.

[19] S.T. Dubas, T.R. Farhat, J.B. Schlenoff, Multiple membranes from "true" polyelectrolyte multilayers, J. Am. Chem. Soc. 123 (2001) 5368-5369.

[20] H.W. Jomaa, J.B. Schlenoff, Salt-induced polyelectrolyte interdiffusion in multilayered films: a neutron reflectivity study, Macromolecules 38 (2005) 8473-8480.

[21] S.E. Burke, C.J. Barrett, Acid-base equilibria of weak polyelectrolyte in multilayer thin films, Langmuir 19 (2003) 3297-3303.

[22] J.B. Schlenoff, S.T. Dubas, Mechanism of polyelectrolyte multilayer growth charge overcompensation and distribution, Macromolecules 34 (3) (2001) 592-598.

[23] S.S. Shiratori, M.F. Rubner, pH-Dependent thickness behavior of sequentially adsorbed layers of weak polyelectrolytes, Macromolecules 33 (2000) 4213-4219.

[24] J.L Lutkenhaus, K. McEnnis, P.T. Hammond, Nano- and microporous layer-bylayer assemblies containing linear poly(ethyleneimine) and poly(acrylic acid), Macromolecules 41 (2008) 6047-6054.

[25] A.I. Petrov, A.A. Antipov, G.B. Sukhorukov, Base-acid equilibria in polyelectrolyte system: from weak polyelectrolytes to interpolyelectrolyte complexes and multilayered polyelectrolyte shells, Macromolecules 36 (2003) 10079-10086.

[26] J. Choi, M.F. Rubner, Influence of the degree of ionization on weak polyelectrolyte multilayer assembly, Macromolecules 38 (2005) 116-124.

[27] H. Zhang, D. Wang, Z. Wang, X. Zhang, Hydrogen bonded layer-by-layer assembly of poly(2-vinylpyridine) and poly(acrylic acid): influence of molecular weight on the formation of microporous film by post-base treatment, Eur. Polym. J. 43 (2007) 2784-2791.

[28] X. Zhang, T. Wu, J. Sun, J. Shen, Ways for fabricating stable layer-by-layer selfassemblies: combined ionic self-assembly and post chemical reaction, Colloids Surf. A 198-200 (2002) 439-442.

[29] J. Dejeu, S. Diziain, C. Dange, F. Membrey, D. Charraut, A. Foissy, Stability of selfassembled polymer films investigated by optical laser reflectometry, Langmuir 24 (2008) 3090-3098.

[30] A.F. Xie, S. Granick, Local electrostatics within a polyelectrolyte multilayer with embedded weak polyelectrolyte, Macromolecules 35 (2002) 1805-1813.

[31] G. Zhang, N. Wang, X. Song, S. Ji, Z. Liu, Preparation of pilot-scale inner skin hollow fiber pervaporation membrane module: effects of dynamic assembly conditions, J. Membr. Sci. 338 (2009) 43-50.

[32] G. Zhang, X. Song, S. Ji, N. Wang, Z. Liu, Self-assembly of inner skin hollow fiber polyelectrolyte multilayer membranes by a dynamic negative pressure layer-by-layer technique, J. Membr. Sci. 325 (2008) 109-116.

[33] Z. Liu, G. Zhang, Y. Peng, S. Ji, The hollow fiber ultrafiltration membrane with inner skin and its application, Desalination 233 (2008) 55-63.

[34] D. Yoo, S.S. Shiratori, M.F. Rubner, Controlling bilayer composition and surface wettability of sequentially adsorbed multilayers of weak polyelectrolytes, Macromolecules 31 (1998) 4309-4318.

[35] J.D. Mendelsohn, C.J. Barrett, V.V. Chan, A.J. Pal, A.M. Mayes, M.F. Rubner, Fabrication of microporous thin films from polyelectrolyte multilayers, Langmuir 16 (2000) 5017-5023.

[36] N. Kato, P. Schuetz, A. Fery, F. Caruso, Thin multilayer films of weak polyelectrolytes on colloid particles, Macromolecules 35 (26) (2002) 9780-9787.

[37] J.J. Harris, P.M. DeRose, M.L. Bruening, Synthesis of passivating, nylon-like coatings through cross-linking of ultrathin polyelectrolyte films, J. Am. Chem. Soc. 121 (9) (1999) 1978-1979.

[38] J.H. Kim, S.Y. Ha, S.Y. Nam, J.W. Rhim, K.H. Baek, Y.M. Lee, Selective permeation of $\mathrm{CO}_{2}$ through pore-filled polyacrylonitrile membrane with poly(ethylene glycol), J. Membr. Sci. 186 (2001) 97-107.

[39] F. Caruso, H. Mohwald, Protein multilayer formation on colloids through a stepwise self-assembly technique, J. Am. Chem. Soc. 121 (1999) 6039-6046. 